\title{
The Annihilating-Ideal Graph of Commutative Rings I*
}

\author{
M. Behboodi ${ }^{a, b \dagger}$ and Z. Rakeei ${ }^{a}$ \\ ${ }^{a}$ Department of Mathematical Science, Isfahan University of Technology, \\ P. O. Box: 84156-83111, Isfahan, Iran \\ ${ }^{b}$ School of Mathematics, Institute for Research in Fundamental Sciences (IPM), \\ P. O. Box: 19395-5746, Tehran, Iran \\ mbehbood@cc.iut.ac.ir, sanamzhr@yahoo.com
}

\begin{abstract}
Let $R$ be a commutative ring with $\mathbb{A}(R)$ its set of ideals with nonzero annihilator. In this paper and its sequel, we introduce and investigate the annihilatingideal graph of $R$, denoted by $\mathbb{A} \mathbb{G}(R)$. It is the (undirected) graph with vertices $\mathbb{A}(R)^{*}:=\mathbb{A}(R) \backslash\{(0)\}$, and two distinct vertices $I$ and $J$ are adjacent if and only if $I J=(0)$. First, we study some finiteness conditions of $\mathbb{A} \mathbb{G}(R)$. For instance, it is shown that if $R$ is not a domain, then $\mathbb{A} \mathbb{G}(R)$ has ACC (resp., DCC) on vertices if and only if $R$ is Noetherian (resp., Artinian). Moreover, the set of vertices of $\mathbb{A} \mathbb{G}(R)$ and the set of nonzero proper ideals of $R$ have the same cardinality when $R$ is either an Artinian or a decomposable ring. This yields for a ring $R, \mathbb{A} \mathbb{G}(R)$ has $n$ vertices $(n \geq 1)$ if and only if $R$ has only $n$ nonzero proper ideals. Next, we study the connectivity of $\mathbb{A} \mathbb{G}(R)$. It is shown that $\mathbb{A G}(R)$ is a connected graph and $\operatorname{diam}(\mathbb{A} \mathbb{G})(R) \leq 3$ and if $\mathbb{A} \mathbb{G}(R)$ contains a cycle, then $\operatorname{gr}(\mathbb{A} \mathbb{G}(R)) \leq 4$. Also, rings $R$ for which the graph $\mathbb{A G}(R)$ is complete or star, are characterized, as well as rings $R$ for which every vertex of $\mathbb{A} \mathbb{G}(R)$ is a prime (or maximal) ideal. In Part II we shall study the diameter and coloring of annihilating-ideal graphs.
\end{abstract}

Key Words: Commutative rings; Annihilating-ideal; Zero-divisor; Graph

2000 Mathematics Subject Classification: 13A15; 05C75.

${ }^{*}$ The research of the first author was in part supported by a grant from IPM (No. 87160026).

${ }^{\dagger}$ Corresponding author. 


\section{Introduction}

In the literature, there are many papers on assigning a graph to a ring, a group, semigroup or a module (see for example [1-11,13-17]). In fact, the concept of the zero divisor graph of a commutative ring $R$ was first introduced by Beck [6], where he was mainly interested in colorings. In his work all elements of the ring were vertices of the graph. This investigation of colorings of a commutative ring was then continued by Anderson and Naseer in [2]. Let $Z(R)$ be the set of zero-divisors of $R$. In [3], Anderson and Livingston associate a graph, $\Gamma(R)$, to $R$ with vertices $Z(R) \backslash\{0\}$, the set of nonzero zero-divisors of $R$, and for distinct $x, y \in Z(R) \backslash\{0\}$, the vertices $x$ and $y$ are adjacent if and only if $x y=0$. In [18], Sharma and Bhatwadekar define another graph on $R, G(R)$, with vertices as elements of $R$, where two distinct vertices $a$ and $b$ are adjacent if and only if $R a+R b=R$ (see also [15], in which, the notion of "comaximal graph of commutative rings" is investigated). Recently Anderson and Badawi in [4] have introduced and investigated the total graph of $R$, denoted by $T(\Gamma(R))$. It is the (undirected) graph with all elements of $R$ as vertices, and for distinct $x, y \in R$, the vertices $x$ and $y$ are adjacent if and only if $x+y \in Z(R)$.

In ring theory, the structure of a ring $R$ is closely tied to ideal's behavior more than elements', and so it is deserving to define a graph whose vertices are ideals instead of elements.

Throughout this paper $R$ will be a commutative ring with identity. We denote the set of all proper ideals of $R$ by $\mathbb{I}(R)$. We name an ideal $I$ of $R$, an annihilating-ideal if there exists a nonzero ideal $J$ of $R$ such that $I J=(0)$, and use the notation $\mathbb{A}(R)$ for the set of all annihilating-ideals of $R$. Also $\operatorname{Spec}(R), \operatorname{Max}(R)$ and $J(R)$ denote the sets of prime ideals, maximal ideals and the Jacobson radical of $R$, respectively.

In this paper, we define annihilating-ideal graph of $R$, denoted by $\mathbb{A} \mathbb{G}(R)$, as a graph with vertices $\mathbb{A}(R)^{*}=\mathbb{A}(R) \backslash\{(0)\}$, where distinct vertices $I$ and $J$ are adjacent if and only if $I J=(0)$. We investigate the interplay between the graph-theoretic properties of $\mathbb{A} \mathbb{G}(R)$ and the ring-theoretic properties of $R$. In Section 1, we study some finiteness conditions of annihilating-ideal graphs. For instance, it is shown that if $R$ is not a domain, then $\mathbb{A} G(R)$ has assenting chain condition $(A C C)$ (resp., descending chain condition $(D C C)$ ) on vertices if and only if $R$ is a Noetherian (resp., an Artinian) ring. Also, it is shown that $\mathbb{A G}(R)$ has $n$ vertices $(n \geq 1)$ if and only if $R$ has only $n$ nonzero proper ideals. These facts motivates us to the following conjecture: for a non-domain ring $R$, the set of vertices of $\mathbb{A} \mathbb{G}(R)$ and the set of nonzero proper ideals of $R$ have the same cardinality. The conjecture above is true 
for all Artinian rings as well as all decomposable rings (see Proposition 1.3 and Proposition 1.6).

Recall that a graph $G$ is connected if there is a path between every two distinct vertices. For distinct vertices $x$ and $y$ of $G$, let $d(x, y)$ be the length of the shortest path from $x$ to $y$ and if there is no such path we define $d(x, y)=\infty$. The diameter of $G$ is $\operatorname{diam}(G)=\sup \{d(x, y)$ : $x$ and $y$ are distinct vertices of $G$. The girth of $G$, denoted by $\operatorname{gr}(G)$, is defined as the length of the shortest cycle in $G$ and $\operatorname{gr}(G)=\infty$ if $G$ contains no cycles. A graph in which each pair of distinct vertices is joined by an edge is called a complete graph. Also, if a graph $G$ contains one vertex to which all other vertices are joined and $G$ has no other edges, is called a star graph. In Section 2, the connectivity of the annihilating-ideal graphs are studied. It is shown that for every ring $R, \mathbb{A G}(R)$ is a connected graph and $\operatorname{diam}(\mathbb{A} \mathbb{G})(R) \leq 3$, and if $\mathbb{A} \mathbb{G}(R)$ contains a cycle, then $\operatorname{gr}(\mathbb{A} \mathbb{G}(R)) \leq 4$. Also, rings $R$ for which the graph $\mathbb{A} \mathbb{G}(R)$ is a complete (or star) graph are characterized, as well as rings $R$ for which every vertex of $\mathbb{A} G(R)$ is a prime (or maximal) ideal. In part II we shall continue the study of this construction via diameter and coloring.

\section{Finiteness conditions of annihilating-ideal graphs}

For a ring $R, \operatorname{soc}(R)$ is the sum of all minimal ideals of $R$ (if there are no minimal ideals, this sum is defined to be zero). Also, if $X$ is an element or a subset of a ring $R$, we define the annihilator of $X$ in $R$ by $\operatorname{Ann}(X)=\{r \in R \mid r X=(0)\}$. An ideal $I$ of a $\operatorname{ring} R$ is called an annihilator ideal if $I=A n n(x)$ for some $x \in R$. Also, for a set $A$ we denote the cardinal number of $A$ by $|A|$.

Let $R$ be a ring. We say that the annihilating-ideal graph $\mathbb{A} \mathbb{G}(R)$ has $A C C$ (resp., $D C C$ ) on vertices if $R$ has $A C C$ (resp., $D C C$ ) on $\mathbb{A}^{*}(R)$.

Theorem 1.1. Let $R$ be a non-domain ring. Then $\mathbb{A} \mathbb{G}(R)$ has $A C C$ (resp., $D C C$ ) on vertices if and only if $R$ is a Noetherian (resp., an Artinian) ring.

Proof. Suppose that $\mathbb{A} \mathbb{G}(R)$ has $A C C$ (resp., $D C C$ ) on vertices. Let $0 \neq$ $x \in Z(R)$ and $P=\operatorname{Ann}(x)$. Then

$$
\{I: I \unlhd R, I \subseteq R x\} \bigcup\{I: I \unlhd R, I \subseteq P\} \subseteq \mathbb{A}(R) .
$$

It follows that the $R$-modules $R x$ and $P$ have $A C C$ (resp., $D C C$ ) on sub- 
modules i.e., $R x$ and $P$ are Noetherian (resp., Artinian) $R$-modules. Since $R x \cong R / P$, by [19, Proposition 7.17], $R$ is a Noetherian (resp., an Artinian) ring. The converse is clear.

In view of above theorem and Cohen's theorem, one may naturally ask, when $\operatorname{Spec}(R) \cap \mathbb{A}(R)^{*} \neq \emptyset$ and every prime ideal $P \in \mathbb{A}(R)^{*}$ is finitely generated, is $R$ Noetherian? The answer is no! The following example gives a non-Noetherian ring $R$ for which every prime vertex of $\mathbb{A} \mathbb{G}(R)$ is finitely generated.

Example 1.2. Let $K$ be a field and $D:=K\left[\left\{x_{i}: i \in \mathbb{Z}\right\}\right]$. The domain $D$ is a unique factorization domain and $Q=x_{1} D$ is a principal height one prime of $D$. Let $R:=D / x_{1}^{2} D$ and $P_{0}:=Q / x_{1}^{2} D$. Then $P_{0}$ is a prime ideal of $R$ with $P_{0}^{2}=(0)$. Thus $P_{0} \in \mathbb{A} G(R)$ and $P_{0} \subseteq P$ for every $P \in \operatorname{Spec}(R)$. Moreover, $x_{1}^{2} D$ is a $Q$-primary ideal of $D$. It follows that the zero ideal of $R$ is $P_{0}$-primary. Hence $I J=(0)$ in $R$ with $I \neq(0)$ and $J \neq(0)$, implies both $I$ and $J$ are contained in $P_{0}=Q / x_{1}^{2} D$. Therefore, $P_{0}=Z(R)$ is a cyclic ideal and $\operatorname{Spec}(R) \cap \mathbb{A}(R)^{*}=\left\{P_{0}\right\}$, but $R$ is not a Noetherian ring.

The following proposition shows that rings $R$ for which every nonzero proper ideal $I$ of $R$ is a vertex of $\mathbb{A} \mathbb{G}(R)$ are abundant.

Proposition 1.3. Let $R$ be an Artinian ring. Then every nonzero proper ideal $I$ of $R$ is a vertex of $\mathbb{A} \mathbb{G}(R)$.

Proof. Let $R$ be an Artinian ring. Then each maximal ideal has a nonzero annihilator (since each is also a minimal prime, and a minimal prime in a Noetherian ring always has a nonzero annihilator). Thus every nonzero proper ideal $I$ of $R$ is a vertex of $\mathbb{A} \mathbb{G}(R)$.

The following result shows that the set of all rings for which the annihilatingideal graphs are finite coincides with the set of all (Artinian) rings with only finitely many proper ideals. Moreover, for a ring $R$, the graph $\mathbb{A} \mathbb{G}(R)$ has $n$ vertices $(n \geq 1)$ if and only if $R$ has only $n$ nonzero proper ideals.

Theorem 1.4. Let $R$ be a ring. Then the following statements are equivalent.

(1) $\mathbb{A} \mathbb{G}(R)$ is a finite graph.

(2) $R$ has only finitely many ideals. 
(3) Every vertex of $\mathbb{A} \mathbb{G}(R)$ has finite degree.

Moreover, $\mathbb{A} \mathbb{G}(R)$ has $n(n \geq 1)$ vertices if and only if $R$ has only $n$ nonzero proper ideals.

Proof. $(1) \Rightarrow(2)$. Suppose that $\mathbb{A} \mathbb{G}(R)$ is a finite graph with $n(n \geq 1)$ vertices. Then by Theorem 1.1, $R$ is an Artinian ring. Now by Proposition 1.3 , every nonzero proper ideal $I$ of $R$ is a vertex of $\mathbb{A} \mathbb{G}(R)$. Thus $\left|\mathbb{I}(R)^{*}\right|=n$ i.e., $R$ has only $n$ nonzero proper ideals.

$(2) \Rightarrow(3)$. Clear.

$(3) \Rightarrow(1)$. Suppose that every vertex of $\mathbb{A} \mathbb{G}(R)$ has finite degree, but $\mathbb{A} \mathbb{G}(R)$ is an infinite graph. Let $I=R x$ be a vertex of $\mathbb{A G}(R)$ and $J=A n n(I)$. If the set of $R$-submodules of $I$ (resp., $J$ ) is infinite, then $J$ (resp., $I$ ) has infinite degree, a contradiction. Thus the set of $R$-submodules of $I$ and $J$ are finite and hence $I$ and $J$ are Artinian $R$-module. Now, since $I \cong R / J$, $R$ is an Artinian ring. Thus by Proposition 1.3, every nonzero proper ideal of $R$ is a vertex of $\mathbb{A} \mathbb{G}(R)$. We consider the following 2 cases:

Case 1: $R$ is a local ring. Since $I$ has finite number of $R$-submodules, $R$ has a minimal ideal, say $R x_{0}$. Let $P=\operatorname{Ann}\left(x_{0}\right)$. Then $P$ is the maximal ideal of $R$ and so every proper ideal of $R$ is contained in $P$. This implies that $R x_{0}$ is adjacent to all other vertices of $\mathbb{A} \mathbb{G}(R)$. Since $R x_{0}$ has finite degree, $\mathbb{A} \mathbb{G}(R)$ is a finite graph.

Case 2: $R$ is not local. Suppose that $R=R_{1} \times R_{2}$ where $R_{1}$ and $R_{2}$ are nonzero rings. Then for each ideal $I$ of $R_{1}$ the vertex $I \times(0)$ of $\mathbb{A} \mathbb{G}(R)$ is adjacent to $(0) \times R_{2}$. Thus the set of ideals of $R_{1}$ is finite. Similarly, the set of ideals of $R_{2}$ is finite. This implies that the set of ideals of $R$ is finite, i.e., $\mathbb{A} \mathbb{G}(R)$ is a finite graph.

Finally, by Proposition 1.3 , we conclude that $\mathbb{A} \mathbb{G}(R)$ has $n(n \geq 1)$ vertices if and only if $R$ has only $n$ nonzero proper ideals.

We have not found any examples of a non-domain ring $R$ such that $\left|\mathbb{A}^{*}(R)\right|<\left|\mathbb{I}^{*}(R)\right|$. The lack of such counterexamples, together with the fact that $\left|\mathbb{A}^{*}(R)\right|=\left|\mathbb{I}^{*}(R)\right|$ where $R$ is an Artinian ring (see Proposition 1.3 and Theorem 1.4) or $R$ is a decomposable ring (see Proposition 1.6), motivates the following fundamental conjecture:

Conjecture 1.5. Let $R$ be a non-domain ring. Then the set of vertices of $\mathbb{A} \mathbb{G}(R)$ and the set of nonzero proper ideals of $R$ have the same cardinality.

Proposition 1.6. The Conjecture 1.5 is true for each decomposable ring $R$. 
Proof. Suppose that $R=R_{1} \times R_{2}$. If both $R_{1}$ and $R_{2}$ are Artinian i.e., $R$ is an Artinian ring, then by Proposition 1.3, the proof is complete. Thus we can assume that $R_{1}$ is not Artinian and so the set of nonzero proper ideals of $R_{1}$ is infinite. Clearly, every ideal of $R$ is of the form $I_{1} \times I_{2}$ where $I_{1}$ and $I_{2}$ are ideals of $R_{1}$ and $R_{2}$, respectively. Note that $\left|\mathbb{I}\left(R_{1}\right) \times \mathbb{I}\left(R_{2}\right)\right|$ equals $\max \left\{\left|\mathbb{I}\left(R_{1}\right)\right|,\left|\mathbb{I}\left(R_{2}\right)\right|\right\}$. Also every ideal of the form $I \times(0)$ or $(0) \times J$, where $I$ is an ideal of $R_{1}$ and $J$ is an ideal of $R_{2}$, is a vertex of $\mathbb{A} \mathbb{G}(R)$. Clearly $\left|\left\{I \times(0): I \in \mathbb{I}\left(R_{1}\right)\right\}\right|=\left|\mathbb{I}\left(R_{1}\right)\right|$ and $\left|\left\{(0) \times J: J \in \mathbb{I}\left(R_{2}\right)\right\}\right|=\left|\mathbb{I}\left(R_{2}\right)\right|$ and hence

$$
|\mathbb{A}(R)| \geq \max \left\{\left|\mathbb{I}\left(R_{1}\right)\right|,\left|\mathbb{I}\left(R_{2}\right)\right|\right\}=\left|\mathbb{I}\left(R_{1}\right)\right| \times\left|\mathbb{I}\left(R_{2}\right)\right|=|\mathbb{I}(R)| .
$$

On the other hand $|\mathbb{A}(R)| \leq|\mathbb{I}(R)|$. It follows that $\left|\mathbb{A}(R)^{*}\right|=\left|\mathbb{I}(R)^{*}\right|$.

The following result shows that if every nonzero proper ideal of a Noetherian ring $R$ is a vertex of $\mathbb{A} \mathbb{G}(R)$, then $R$ is a semilocal ring (i.e., $R$ has only finitely many maximal ideals).

Proposition 1.7. Let $R$ be a Noetherian ring. If all nonzero proper ideals of $R$ are vertices of $\mathbb{A G}(R)$, then $R$ is a semilocal ring.

Proof. Suppose that $\left\{P_{n} \mid n \in \mathbb{N}\right\}$ are distinct maximal ideals of $R$. Then by our assumption, $\operatorname{Ann}\left(P_{n}\right) \neq(0)$ for each $n \geq 1$. It follows that for each $n$ there exists $x_{n} \in R$ such that $P_{n}=\operatorname{Ann}\left(x_{n}\right)$. Thus for each $n, x_{n}$ is in every maximal ideal $P_{k}$ for $k \neq n$. By ACC, the chain $R x_{1} \varsubsetneqq R x_{1}+R x_{2} \varsubsetneqq \cdots \varsubsetneqq R x_{1}+\cdots+R x_{n} \varsubsetneqq \cdots$ must stabilize, and each step is proper since $P_{1} \cap P_{2} \cap \cdots \cap P_{k}$ is the annihilator of $x_{1} R+x_{2} R+\cdots+x_{k} R$ for each $k$. Therefore, $R$ has only finitely many maximal ideals, i.e., $R$ is semilocal.

The following result shows that for each Noetherian ring $R$, if $R$ is not a domain, then at least one of the vertices of $\mathbb{A} \mathbb{G}(R)$ is a prime ideal.

Proposition 1.8. Let $R$ be a Noetherian ring. Then either $\mathbb{A}(R)^{*}=\emptyset$ or $\operatorname{Spec}(R) \cap \mathbb{A}(R)^{*} \neq \emptyset$.

Proof. Assume $\mathbb{A}(R)^{*} \neq \emptyset$ i.e., $R$ is not a domain. Clearly, the set of all annihilators of nonzero elements of $R$ is a subset of $\mathbb{A}(R)^{*}$. On the other hand by our hypothesis there exists an annihilator ideal $P$ of $R$ which is 
maximal among all annihilators of nonzero elements of $R$. Now by [13, Theorem 6], $P$ is a prime ideal. Since $R$ is not a domain, $P \neq(0)$ and so $P \in \operatorname{Spec}(R) \cap \mathbb{A}(R)^{*}$.

The following example shows that the Noetherian hypothesis is needed in Proposition 1.8.

Example 1.9. Let $K$ be a field, $D:=K\left[\left\{x_{i}: i \in \mathbb{N}\right\}\right]$ (a unique factorization domain) and $R=K\left[\left\{x_{i}: i \in \mathbb{N}\right\}\right] /\left(\left\{x_{i}^{2}: i \in \mathbb{N}\right\}\right)$. Let $\bar{x}_{i}=x_{i}+\left(\left\{x_{i}^{2}: i \in \mathbb{N}\right\}\right)$ for each $i \in \mathbb{N}$ and $M=\left(\left\{\bar{x}_{i}: i \in \mathbb{N}\right\}\right)$. The ideal $M$ is simply the image of the maximal ideal $N=\left(\left\{x_{i}: i \in \mathbb{N}\right\}\right)$ of $D$. Moreover $N$ is the radical of $J=\left(\left\{x_{i}^{2}: i \in \mathbb{N}\right\}\right)$. Thus $R$ is a local zero-dimensional ring (but is not Noetherian). For each $x_{i}$, the conductor of $x_{i}$ into $J$ is the height one (principal) prime $x_{i} D$ (since $x_{i}$ is an irreducible=prime element of $D$ ). Thus viewed as an element of $R$, the annihilator of $x_{i}$ is $x_{i} R$. Obviously $\bigcap x_{i} D=(0)$ and this implies $\bigcap x_{i} R=(0)$. Hence $\operatorname{Ann}(M)=(0)$. Thus $\operatorname{Spec}(R) \cap \mathbb{A}(R)^{*}=\emptyset$.

We need the following two lemmas.

Lemma 1.10. Let $R$ be a ring and $P$ be a maximal ideal such that $P \in$ $\mathbb{A}(R)^{*}$. Then $P=\operatorname{Ann}(x)$ for some $0 \neq x \in R$.

Proof. Let $P \in \mathbb{A}(R)^{*}$ be a maximal ideal of $R$. Then there exists $I \in \mathbb{A}^{*}(R)$, such that $I P=(0)$. Let $0 \neq a \in I$. Then $a P=(0)$ and so $P \subseteq \operatorname{Ann}(a)$. Since $P$ is maximal, $P=\operatorname{Ann}(a)$.

Lemma 1.11. Let $R$ be a ring such that $R$ is not a field. Then for each minimal ideal $I$ of $R, I$ and $A n n(I)$ are vertices of $\mathbb{A} \mathbb{G}(R)$.

Proof. Suppose that $I$ is a minimal ideal of $R$. Since $R$ is not a field, $I \neq R$. Now by Brauer's Lemma (see $[13,10.22]$ ), either $I^{2}=(0)$ or $I=R e$ for some idempotent $1 \neq e \in R$. If $I^{2}=(0)$, then $I$ is a vertex of $\mathbb{A} \mathbb{G}(R)$. If $I^{2}=R e$, then $R=R e \oplus R(1-e)$. Clearly $R e R(1-e)=(0)$ and so $R e$ is a vertex of $\mathbb{A} \mathbb{G}(R)$.

Proposition 1.8, is not true in general, if we replace $\operatorname{Spec}(R)$ with $\operatorname{Max}(R)$. For instance, if $R=\mathbb{Z} \times \mathbb{Z}$, though $R$ has $A C C$ on its ideals and $\mathbb{A}(R)^{*} \neq \emptyset$, we have $\operatorname{Max}(R) \cap \mathbb{A}(R)=\emptyset$. In the following result we characterize all non-domain rings $R$ for which $\operatorname{Max}(R) \cap \mathbb{A}(R) \neq \emptyset$. 
Proposition 1.12. Let $R$ be a non-domain ring. Then $\operatorname{Max}(R) \cap \mathbb{A}(R)^{*} \neq$ $\emptyset$ if and only if $\operatorname{soc}(R) \neq(0)$.

Proof. Suppose that $P \in \operatorname{Max}(R) \cap \mathbb{A}(R)^{*}$. Then by Lemma 1.10, $P=$ $\operatorname{Ann}(x)$ for some $0 \neq x \in R$. Since $R / P \cong R x, R x$ is a minimal ideal of $R$, i.e., $\operatorname{soc}(R) \neq(0)$. Conversely, suppose that $\operatorname{soc}(R) \neq(0)$ and $I$ is a minimal ideal of $R$. Then $I=R x$ for some $x \in I$. Since $R / A n n(x) \cong R x$ and $R x$ is a minimal ideal of $R, \operatorname{Ann}(x)$ is a maximal ideal of $R$ and so $\operatorname{Max}(R) \cap \mathbb{A}(R)^{*} \neq \emptyset$.

We conclude this section with the following result, that gives us a characterization for rings $R$ for which every nonzero proper cyclic ideal $I$ of $R$ is a vertex of $\mathbb{A} \mathbb{G}(R)$. The proof is trivial and left to reader.

Proposition 1.13. Let $R$ be a ring. Then very nonzero proper cyclic ideal of $R$ is a vertex of $\mathbb{A G}(R)$ if and only if every element in $R$ is a unit or a zero-divisor.

\section{Connectivity of the annihilating-ideal graphs}

By Anderson and Livingston [3, Theorem 2.3], for every ring $R$, the zero divisor graph $\Gamma(R)$ is a connected graph and $\operatorname{diam}(\Gamma(R)) \leq 3$. Moreover, if $\Gamma(R)$ contains a cycle, then $\operatorname{gr}(\Gamma(R)) \leq 4$ (see [16]). These facts later were developed by Behboodi [8] for modules over a commutative ring, by Redmond [17], for the undirected zero-divisor graph of a non-commutative ring and by Behboodi and Beyranvand [7] for the strong zero divisor graphs of non-commutative rings. Here we will show that these facts are also true for the annihilating-ideal graph of a ring.

Let $S$ be a commutative multiplicative semigroup with $0(0 x=0$ for all $x \in S$ ). The zero-divisor graph of $S$ (denoted by $\Gamma(S)$ ) is a graph whose vertices are the nonzero zero-divisors of $S$, with two distinct vertices $a, b$ joined by an edge in case $a b=0$. In [11, Theorem 1.2], it is shown that $\Gamma(S)$ is always connected, and $\operatorname{diam}(\Gamma(S)) \leq 3$. Next, we use this to obtain the same result for the annihilating-ideal graph of a ring.

Theorem 2.1. For every ring $R$, the annihilating-ideal graph $\mathbb{A} \mathbb{G}(R)$ is connected and $\operatorname{diam}(\mathbb{A G}(R)) \leq 3$. Moreover, if $\mathbb{A} \mathbb{G}(R)$ contains a cycle, then $\operatorname{gr}(\mathbb{A} \mathbb{G}(R)) \leq 4$. 
Proof. Let $R$ be a ring. Clearly, the set $S:=\mathbb{A}(R)$ is a commutative semigroup under multiplication and also $\mathbb{A} \mathbb{G}(R)=\Gamma(S)$. Thus by $[11$, Theorem 1.2 ], $\mathbb{A} \mathbb{G}(R)$ is a connected graph and $\operatorname{diam}(\mathbb{A G}(R)) \leq 3$.

Now, suppose that $\mathbb{A} \mathbb{G}(R)$ contains a cycle, and let $C:=I_{1}-\ldots-I_{n}-I_{1}$ be a cycle with the least length. If $n \leq 4$, we are done. Otherwise, we have $I_{1} \cap I_{4} \neq(0)$. We need only consider 3 cases:

Case 1: $I_{1} \cap I_{4}=I_{1}$. Then $I_{1} I_{3} \subseteq I_{4} I_{3}=(0)$ and $I_{1}-I_{2}-I_{3}-I_{1}$ is a cycle, a contradiction. The case $I_{1} \cap I_{4}=I_{4}$ is similar.

Case 2: $I_{1} \cap I_{4}=I_{2}$. Then $I_{2} \subseteq I_{1}, I_{2} I_{n}=(0)$ and so $I_{2}-\ldots-I_{n}-I_{2}$ is a cycle with length $n-1$, a contradiction. The case $I_{1} \cap I_{4}=I_{3}$ is similar. Case 3: $I_{1} \cap I_{4} \neq I_{1}, I_{2}, I_{3}, I_{4}$. Then we have $I_{2}\left(I_{1} \cap I_{4}\right)=(0), I_{3}\left(I_{1} \cap I_{4}\right)=$ $(0)$, and $I_{2}-\left(I_{1} \cap I_{4}\right)-I_{3}-I_{2}$ is a cycle, a contradiction.

Thus $n \leq 4$, i.e., $\operatorname{gr}(\mathbb{A} \mathbb{G}(R)) \leq 4$.

Next, we characterize all rings $R$ for which the graph $\mathbb{A} \mathbb{G}(R)$ has a vertex adjacent to every other vertex. Then we apply this to characterize rings $R$ for which the graph $\mathbb{A} \mathbb{G}(R)$ is complete or star.

Theorem 2.2. Let $R$ be a ring. Then there is a vertex of $\mathbb{A} \mathbb{G}(R)$ which is adjacent to every other vertex if and only if either $R=F \oplus D$, where $F$ is a field and $D$ is an integral domain, or $Z(R)$ is an annihilator ideal.

Proof. $(\Rightarrow)$. Suppose that $Z(R)$ is not an annihilator ideal and the vertex $I_{0} \in \mathbb{A}^{*}(R)$ is adjacent to every other vertex. Let $0 \neq a \in I_{0}$. Then $R a$ is also adjacent to every other vertex. Now $a \notin A n n(a)=I$, for otherwise $Z(R)$ would be an annihilator ideal. If $R b$ is a nonzero ideal of $R$ such that $R b \varsubsetneqq R a$, then $R b$ is also adjacent to every other vertex and also $R b R b \subseteq R b R a=(0)$. Hence for each $x \in Z(R), R x$ is a vertex of $\mathbb{A} \mathbb{G}(R)$ and $(R x)(R b)=(0)$ i.e., $Z(R)=A n n(b)$, a contradiction. Thus $R a$ is a minimal ideal of $R$ with $(R a)^{2} \neq(0)$. Thus by Brauer's Lemma (see [13, 10.22]), $R a=R e$ for some idempotent $e \in R$. So $R=R e \oplus R(1-e)$ and hence we may assume that $R=R_{1} \times R_{2}$ with $R_{1} \times(0)$ adjacent to every other vertex. For each $0 \neq c \in R_{1}, R_{1} c \times(0)$ is an annihilating-ideal of $R$. If $R_{1} c \neq R_{1}$, then $\left(R_{1} \times(0)\right)\left(R_{1} c \times(0)\right)=(0) \times(0)$, i.e., $c R_{1}=(0)$, a contradiction. Thus $R_{1}$ must be a field. If $R_{2}$ is not an integral domain, then there is a nonzero $b \in Z\left(R_{2}\right)$. Then $R_{1} \times R_{2} b$ is an annihilating-ideal of $R$ which is not adjacent to $R_{1} \times(0)$, a contradiction. Thus $R_{2}$ must be an integral domain.

$(\Leftarrow)$. If $R=F \oplus D$, where $F$ is a field and $D$ is an integral domain, then $F \times(0)$ is adjacent to every other vertex. If $Z(R)=A n n(x)$ for some nonzero 
$x \in R$, then $R x$ is adjacent to every other vertex.

Next, we characterize reduced rings $R$ for which the annihilating-ideal graph $\mathbb{A} \mathbb{G}(R)$ is a star graph.

Corollary 2.3. Let $R$ be a reduced ring. Then the following statements are equivalent.

(1) There is a vertex of $\mathbb{A} \mathbb{G}(R)$ which is adjacent to every other vertex.

(2) $\mathbb{A} \mathbb{G}(R)$ is a star graph.

(3) $R=F \oplus D$, where $F$ is a field and $D$ is an integral domain.

Proof. $(1) \Rightarrow(3)$. Suppose there is a vertex of $\mathbb{A} \mathbb{G}(R)$ which is adjacent to every other vertex. If $Z(R)=A n n(x)$ for some $0 \neq x \in R$, then $x^{2}=0$ and so $x=0$, since $R$ is reduced. Thus by Theorem $2.2, R=F \oplus D$, where $F$ is a field and $D$ is an integral domain.

$(3) \Rightarrow(2)$. Let $R=F \oplus D$, where $F$ is a field and $D$ is a domain. Then every nonzero ideal of $R$ is of the form $F \oplus I$ or $(0) \oplus I$ where $I$ is a nonzero proper ideal of $R$. By our hypothesis, and since $\mathbb{A} \mathbb{G}(R)$ is connected, we don't have any vertices of the form $F \oplus I$, such that $I \neq(0)$. Also $F \oplus(0)$ is adjacent to every other vertex, and since $D$ is a domain, non of the ideals of the form $(0) \oplus I$ can be adjacent to each other. So $\mathbb{A} \mathbb{G}(R)$ is a star graph. $(2) \Rightarrow(1)$ is clear.

Corollary 2.4. Let $R$ be a Artinian ring. Then there is a vertex of $\mathbb{A} G(R)$ which is adjacent to every other vertex if and only if either $R=F_{1} \oplus F_{2}$, where $F_{1}, F_{2}$ are fields, or $R$ is a local ring with nonzero maximal ideal.

Proof. $(\Rightarrow)$. Assume there is a vertex of $\mathbb{A} \mathbb{G}(R)$ which is adjacent to every other vertex. Then by Theorem 2.2, either $R=F \oplus D$, where $F$ is a field and $D$ is an integral domain, or $Z(R)$ is an annihilator ideal. Since $R$ is Artinian, if $R=F \oplus D$, then $D$ is an Artinian domain and so $D$ is also a field. Thus $R=F_{1} \oplus F_{2}$, where $F_{1}, F_{2}$ are fields. If $Z(R)$ is an annihilator ideal, then $Z(R)$ is the set of all non-unit elements of $R$ (since $R$ is Artinian). It follows that every maximal ideal of $R$ is contained in $Z(R)$, i.e., $R$ is local with maximal ideal $M=Z(R)$.

$(\Leftarrow)$. If $R=F_{1} \oplus F_{2}$, where $F_{1}, F_{2}$ are fields, then the graph $\mathbb{A} \mathbb{G}(R)$ is a connected graph with two vertices $F_{1} \times(0)$ and $(0) \times F_{2}$. Assume that $R$ is an Artinian local ring with nonzero maximal ideal $M$. Then for each 
minimal ideal $I$ of $R, \operatorname{Ann}(I)=M$, and hence $I$ is a vertex of $\mathbb{A} \mathbb{G}(R)$ which is adjacent to every other vertex.

To have a better characterization for an Artinian ring with star annihilatingideal graph, we need the following Lemma.

Lemma 2.5. Let $R$ be an Artinian ring such that $\mathbb{A} \mathbb{G}(R)$ is a star graph. Then either $R=F_{1} \oplus F_{2}$, where $F_{1}, F_{2}$ are fields, or $R$ is a local ring with nonzero maximal ideal $M$ with $M^{4}=(0)$.

Proof. Let $R$ be an Artinian ring such that $\mathbb{A G}(R)$ is a star graph. Then by Theorem 2.4, either $R=F_{1} \oplus F_{2}$, where $F_{1}, F_{2}$ are fields, or $R$ is a local ring with nonzero maximal ideal $M$. If $R=F_{1} \oplus F_{2}$, there is nothing to prove. Suppose $R$ is a local ring with nonzero maximal ideal $M$. Since $R$ is Artinian, there exists an integer $n \geq 1$ such that $M^{n}=(0)$ and $M^{n-1} \neq(0)$. Clearly $M^{n-1} I \subseteq M^{n-1} M=(0)$, for each ideal $I$ of $R$. Hence $M^{n-1}$ is adjacent to every nonzero ideal $I$ of $R$. If $n>4$, then $M^{2}$ and $M^{n-2}$ will be adjacent, a contradiction. Thus $M(R)^{4}=(0)$.

Theorem 2.6. Let $R$ be an Artinian ring. Then $\mathbb{A G}(R)$ is a star graph if and only if either $R=F_{1} \oplus F_{2}$, where $F_{1}, F_{2}$ are fields, or $R$ is a local ring with nonzero maximal ideal $M$ and one of the following cases holds.

(i) $M^{2}=(0)$ and $M$ is the only nonzero proper ideal of $R$.

(ii) $M^{3}=(0), M^{2}$ is the only minimal ideal of $R$ and for every distinct proper ideals $I_{1}, I_{2}$ of $R$ such that $M^{2} \neq I_{i}(i=1,2), I_{1} I_{2}=M^{2}$.

(iii) $M^{4}=(0), M^{3} \neq(0)$ and $\mathbb{A}^{*}(R)=\left\{M, M^{2}, M^{3}\right\}$.

Proof. $(\Rightarrow)$. By Lemma 2.5, either $R=F_{1} \oplus F_{2}$, where $F_{1}, F_{2}$ are fields, or $R$ is a local ring with nonzero maximal ideal $M$ such that $M^{4}=(0)$.We proceed by the following cases:

Case 1: $M^{2}=(0)$. It follows that $M$ is the vertex adjacent to all other vertices. Assume there are more than one nonzero proper ideals. Then there are nonzero elements $x, y \in M$ with $x R \neq y R$. Since $\mathbb{A} \mathbb{G}(R)$ is a star graph and $M^{2}=(0)$, either $x R=M$ or $y R=M$. Without loss of generality we can assume that $y R=M$. Thus $x R \varsubsetneqq M=y R$ and hence $x=y r$ for some $r \in R$. Since $x R \neq y R, r$ is not a unit element of $R$ and so $r \in M$. It follows that $x=0$, a contradiction. Thus $M$ is the only nonzero proper ideal of $R$. Case 2: $M^{3}=(0)$ and $M^{2} \neq(0)$. Clearly $M^{2}$ is the vertex adjacent to all 
other vertices. Suppose that $I$ is a minimal ideal of $R$ such that $I \neq M^{2}$. Since $\operatorname{Ann}(I)=M, I$ is also adjacent to all other vertices, a contradiction. Thus $M^{2}$ is the only minimal ideal of $R$. Now we assume that $I_{1}$, $I_{2}$ are distinct proper ideals of $R$ such that $M^{2} \neq I_{i}(i=1,2)$. Clearly, $I_{1} I_{2} \subseteq M^{2}$. On the other hand $I_{1} I_{2} \neq(0)$ (since $\mathbb{A} \mathbb{G}(R)$ is a star graph). Hence $I_{1} I_{2}=M^{2}$.

Case 3: $M^{4}=(0)$ and $M^{3} \neq(0)$. Since $\mathbb{A} \mathbb{G}(R)$ is a star graph and $R$ is local, then clearly the center of the star must be a nonzero principal ideal. In the case $M^{3} \neq M^{4}=(0)$, this ideal must be $M^{3}$. Since $M^{2} \supsetneqq M^{3}$, there are elements $a, b \in M \backslash M^{2}$ such that $a b \in M^{2} \backslash M^{3}$. Then $a b M^{2}=(0)$, so the star shaped assumption yields $M^{2}=a b R$. If $a^{2} \in M^{3}$, then $(R a)(a b R)=(0)$, contradicting the star shaped assumption. Hence $M^{2}=a^{2} R$. Another application of the star shaped assumption yields $a^{3} \neq 0$. So $M^{3}=a^{3} R$. For $c \in M \backslash M^{2}, c a^{2} \in a^{3} R \backslash\{0\}$. From this point an elementary argument puts $c \in a R$. Hence $M=a R$ and $\mathbb{A}^{*}(R)=\left\{M, M^{2}, M^{3}\right\}$.

$(\Leftarrow)$ is clear.

Now we are in position to characterize rings with complete annihilatingideal graphs.

Theorem 2.7. Let $R$ be a ring. Then $\mathbb{A} \mathbb{G}(R)$ is a complete graph if and only if $R$ is one of the following three types of rings:

(1) $R=F_{1} \oplus F_{2}$ where $F_{1}, F_{2}$ are fields,

(2) $Z(R)$ is an ideal of $R$ with $Z(R)^{2}=(0)$, or

(3) $R$ is a local ring with exactly two nonzero proper ideals $Z(R)$ and $Z(R)^{2}$.

Proof. Assume that $R$ is a ring for which $\mathbb{A} \mathbb{G}(R)$ is a complete graph. Then, by Theorem 2.2, either $R=F \oplus D$, where $F$ is a field and $D$ is an integral domain, or $Z(R)$ is an annihilator ideal. Suppose that $R=F \oplus D$, where $F$ is a field and $D$ is an integral domain. If $D$ has a nonzero proper ideal $I$, then $(0) \oplus D$ and $(0) \oplus I$ are vertices of $\mathbb{A G}(R)$ which are not adjacent, a contradiction. Thus $D$ doesn't have any nonzero proper ideal and so it is a field. Let $Z(R)$ be an ideal of $R$. A trivial case is $Z(R)^{2}=(0)(\neq Z(R))$, so assume not and let $x, y \in Z(R) \backslash\{0\}$ be such that $x y \neq 0$. Then it must be that $x R=y R$ with $x^{2} \neq 0 \neq y^{2}$. If $z \in Z(R) \backslash\{0\}$ is such that $z R \neq x R$, then $x z=0$ and either $(x+z) R \neq z R$ or $(x+z) R \neq x R$. If the latter, $0=x(x+z)=x^{2}+x z=x^{2}$, a contradiction. Thus $(x+z) R=x R$, $z \in x R$ and $z^{2}=0$ (using $0=z(x+z)=z^{2}$ ). Therefore $x R=Z(R)$ with 
$x^{3}=0\left(\neq x^{2}\right)$, and $x^{2} R=Z(R)^{2}$ is the only other nonzero ideal of $R$. The converse is clear.

Remark 2.8. Let $R$ be a ring. In [3, Theorem 2.8], it is shown the zero divisor graph $\Gamma(R)$ is a complete graph if and only if either $R=\mathbb{Z}_{2} \oplus \mathbb{Z}_{2}$ or $Z(R)$ is an ideal of $R$ and $Z(R)^{2}=(0)$. Thus by above theorem if $\Gamma(R)$ is a complete graph, then $\mathbb{A} \mathbb{G}(R)$ is also a complete graph, but the converse is not true even if $Z(R)$ is an ideal of $R$. For example, for the ring $\mathbb{Z}_{p^{3}}$, where $p$ is a prime number, $\mathbb{A} G(R)$ is a complete graph but $Z(R)^{2} \neq(0)$ and so $\Gamma(R)$ is not a complete graph.

To close above discussions, we ascertain all rings their annihilating-ideal graphs have less than four vertices.

Corollary 2.9. Let $R$ be a ring. Then

(a) $\mathbb{A} \mathbb{G}(R)$ is a graph with one vertex if and only if $R$ has only one nonzero ideal.

(b) $\mathbb{A G}(R)$ is a graph with two vertices if and only if $R=F_{1} \oplus F_{2}$, where $F_{1}, F_{2}$ are fields or $R$ is an Artinian local ring with exactly two nonzero proper ideals $Z(R)$ and $Z(R)^{2}$.

(c) $\mathbb{A G}(R)$ is a graph with three vertices if and only if $R$ is an Artinian local ring with exactly three nonzero proper ideals $Z(R), Z(R)^{2}$ and $Z(R)^{3}$.

Proof. (a) By Theorem 1.4 is clear.

(b) Suppose that $\mathbb{A} \mathbb{G}(R)$ is a graph with two vertices. Since $\mathbb{A} \mathbb{G}(R)$ is connected, then $\mathbb{A} G(R)$ is a complete (or star) graph. Thus by Theorem 2.6(i) and Theorem 2.7, $R$ should be of the form $F_{1} \oplus F_{2}$, or it should be a local ring with exactly two ideals $Z(R)$ and $Z(R)^{2}$. The converse is clear.

(c) Suppose that $\mathbb{A} \mathbb{G}(R)$ is a graph with three vertices. Then by Theorem $1.4, R$ is an Artinian ring with exactly three nonzero proper ideals. Since $\mathbb{A} \mathbb{G}(R)$ is connected, either it is a complete or a star graph. If $\mathbb{A} \mathbb{G}(R)$ is a complete graph, Theorem 2.7, implies that $Z(R)$ is an ideal of $R$ with $Z(R)^{2}=(0)$. If $Z(R)=p R$ for some $p \in R$, then for each $z \in Z(R)$ such that $z R \neq p R, z=p r$ for some non-unit element $R$. Since $Z(R)^{2}=(0)$, $z=0$ and so $Z(R)$ is the only nonzero proper ideal of $R$, a contradiction. Thus $Z(R)$ is not principal. Let $I$ and $J$ be two nonzero proper ideals of $R$ such that $I, J \varsubsetneqq Z(R)$ and $I \neq J$. Then for each $0 \neq z \in Z(R)$, ei- 
ther $R z=I$ or $R z=J$. It follows that $Z(R)=I \cup J$. Since $Z(R)$ is an ideal, $Z(R)=I$ or $Z(R)=J$, a contradiction. Thus $\mathbb{A} \mathbb{G}(R)$ is a star graph. By Theorem 2.6, either $Z(R)^{3}=(0), Z(R)^{2} \neq(0)$ and $R$ has exactly one nonzero proper ideal $I$ such that $Z(R)^{2} \varsubsetneqq I \varsubsetneqq Z(R)$ or $R$ has exactly three nonzero proper ideals $Z(R), Z(R)^{2}$ and $Z(R)^{3}$. In the first case, if $Z(R)$ is not principal, then for each $0 \neq z \in Z(R)$, either $z R=I$ or $z R=Z(R)^{2}$, i.e, $Z(R)=I \bigcup Z(R)^{2}$, a contradiction. Thus $R$ is local with principal maximal ideal $Z(R)=p R$ such that $p^{2} \neq p^{3}=0$. Let $z \in Z(R)$ such that $z R \neq Z(R)$. Then $z=p r$ for some non-unit element $r \in R$ (i.e, $r \in Z(R)$ ). Therefore $z p^{2}=0$, and since $\mathbb{A} \mathbb{G}(R)$ is a star graph, $z R=p^{2} R$. Thus $p^{2} R$ is the only other nonzero proper ideal of $R$, a contradiction. Thus $R$ has exactly three nonzero proper ideals $Z(R), Z(R)^{2}$ and $Z(R)^{3}$. The converse is clear.

A natural question one may ask is "what will happen if all vertices of $\mathbb{A} \mathbb{G}(R)$ are prime (resp., maximal) ideals of $R$ ". That is, what we answered below, in Theorem 2.10. In fact, we characterize all rings satisfy this conditions.

Theorem 2.10. Let $R$ be a ring such that $R$ is not a domain. If every vertex of $\mathbb{A} \mathbb{G}(R)$ is a prime ideal of $R$ (i.e. $\mathbb{A}^{*}(R) \subseteq S p e c(R)$ ), then either either $R=F_{1} \oplus F_{2}$ for a pair of fields $F_{1}$ and $F_{2}$ or $R$ has only one nonzero proper ideal.

Proof. Let $R$ be a ring such that $R$ is not a domain, and suppose that $\left.\mathbb{A}^{*}(R) \subseteq \operatorname{Spec}(R)\right)$. First, if $z$ is a nonzero zero divisor, then $Q=z R$ is a prime ideal. If $Q=Q^{2}$, then can assume $z=z^{2}$. The ideal $N=(1-z) R$ must be a prime as well. Neither $Q$ nor $N$ can have proper subideals. Hence in this case $R=F_{1} \oplus F_{2}$ for fields $F_{1}$ and $F_{2}$. The other possibility is that $x^{2}=0$ for all nonzero $x \in Z(R)$. As $x R$ has to be prime, $x R=Z(R)$ must be the only nonzero proper ideal. [For $r \in R \backslash Z(R), r x R=x R$ which leads to $r$ being a unit.].

The following is now immediate.

Corollary 2.11. Let $R$ be a ring such that it is not a domain. Then the following statements are equivalent.

(1) $\mathbb{A}(R)^{*} \subseteq \operatorname{Max}(R)$, i.e., every vertex of $\mathbb{A} \mathbb{G}(R)$ is a maximal ideal of $R$.

(2) $\mathbb{A}(R)^{*}=\operatorname{Max}(R)$. 
(3) $\mathbb{A}(R)^{*}=\operatorname{Spec}(R)$.

(4) $\mathbb{A}(R)^{*} \subseteq \operatorname{Spec}(R)$.

(5) Either $R=F_{1} \oplus F_{2}$, where $F_{1}, F_{2}$ are fields, or $R$ has only one nonzero proper ideal.

Note: In Part II we shall continue the study of this contraction.

\section{Acknowledgments}

This work was partially supported by the Center of Excellence of Algebraic Methods and Application of Isfahan University of Technology.

\section{References}

[1] S. Akbari, A. Mohammadian, Zero-divisor graphs of non-commutative rings, J. Algebra 296 (2006) 462-479.

[2] D. D. Anderson, M. Naseer, Beck's coloring of a commutative ring, J. Algebra 159 (1993) 500-514.

[3] D. F. Anderson, P. S. Livingston, The zero-divisor graph of a commutative ring, J. Algebra 217 (1999) 434-447.

[4] D. F. Anderson, A. Badawi, The total graph of a commutative ring, J. Algebra in press.

[5] M. Axtell, J. Coykendall, J. Stickles, Zero-divisor graphs of polynomials and power series over rings, Comm. Algebra, 33 (2005) 2043-2050.

[6] I. Beck, Coloring of commutative rings, J. Algebra 116 (1988) 208-226.

[7] M. Behboodi, R. Beyranvand, Strong zero-divisors graphs of non-commutative rings, Int. J. Algebra 2(1) (2008), 25-44.

[5] M. Behboodi, Zero divisor graphs of modules over a commutative rings, J. Commutative Algebra, to appear.

[6] A. Cannon, K. Neuerburg, S.P. Redmond, Zero-divisor graphs of nearrings and semigroups, in: H. Kiechle, A. Kreuzer, M.J. Thomsen (Eds.), Nearrings and Nearfields, Springer, Dordrecht, The Netherlands, 2005, pp. 189-200.

[10] F. DeMeyer, L. DeMeyer, Zero divisor graphs of semigroups, J. Algebra 283 (2005) 190-198.

[11] F. DeMeyer, T. McKenzie and K. Schneider, The zero-divisor graph of a commutative semigroup, Semigroup Forum 65 (2) (2002), 206-214.

[12] I. Kaplansky, Commutative Rings, rev. ed., Univ. of Chicago Press, Chicago, 1974.

[13] T. Y. Lam, A first course in non-commutative rings, Springer-Verlag New York, Inc 1991.

[14] T. G. Lucas, The diameter of a zero divisor graph. J. Algebra 301 (2006) 174-193.

[15] H. R. Maimani, M. Salimi, A. Sattari, S. Yassemi, Comaximal graph of commutative rings, J. Algebra 319 (2008) 1801-1808.

[16] S. B. Mulay, Cycles and symmetries of zero-divisors, Comm. Algebra, 30 (2002) 35333558 .

[17] S.P. Redmond, The zero-divisor graph of a non-commutative ring, Internat. J. Com- 
mutative Rings, 1 (4) (2002) 203-211.

[18] P. K. Sharma, S. M. Bhatwadekar, A note on graphical representation of rings, J. Algebra 176 (1995) 124-127.

[19] R. Y. Sharp, Steps in algebra, Second edition, London Mathematical Society Student Texts, 51. Cambridge University Press, Cambridge, 1990. 